\title{
Influence of dietary fats on circulating amino acid profile in experimental breast cancer
}

\author{
María Jesús Ramírez-Expósito, María Dolores Ruíz-Sanjuan and José Manuel Martínez-Martos* \\ Experimental and Clinical Physiopathology Research Group CTS1039, Department of Health Sciences, School of Experimental and Health Sciences, University of \\ Jaen, Campus Las Lagunillas, E-23071 Jaen, Spain
}

\begin{abstract}
Amino acid levels are tightly regulated through a continuous cycle of protein formation/degradation to maintain neutral protein balance. Tumor cells require a greater amount of amino acids for the synthesis of new proteins and nucleic acids; therefore, changes in the circulating amino acid profile may be detected and could be used to establish a specific profile for each type of cancer. However, the influence of dietary compounds, which could alter these patterns, has been described in cancer. Here we analyzed the effects of several dietary fats [extra virgin olive oil, sunflower oil and sunflower oil enriched with oleic acid] together with standard commercial diet, on serum free amino acids in rats with $\mathrm{N}$-methyl-nitrosourea-induced breast cancer. We examined the changes in circulating free amino acid levels, as well as if a specific amino acid profile existed and whether the type of dietary fat modified this profile. We found changes in several amino acids, including aspartic acid, glutamine, glycine, 1-methyl-histidine, 3-methyl-histidine, ornithine, taurine and alanine, depending on the type of dietary fat used. Our results indicate that the establishment of a specific amino acid profile for breast cancer could be influenced by external factors such as dietary fat.
\end{abstract}

\section{Introduction}

It is well known that circulating free amino acid levels reflect all factors that affect the body's amino acid flow, and are tightly regulated through a continuous cycle of protein formation-degradation in order to maintain neutral protein balance in physiological conditions [1]. However, amino acids also play an important role as metabolic regulators $[2,3]$ of a myriad of processes such as gene expression, immune function and cell growth and differentiation [3-5]. All these processes are involved in some way in tumor development. In fact, it has been extensively described that carcinogenesis is associated with genetic mutations and therefore with an upset gene expression that might produce changes in the glycolitic and lipid metabolism as well as to alterations in amino acids and nucleotides. Thus, the presence of a tumor has frequently been associated with an alteration in protein metabolism and is characterized by an increase in protein exchange [6]. In fact, the tumor cells require a greater amount of amino acids for the synthesis of proteins and nucleic acids [7-9]. Therefore, the circulating free amino acids profile may be altered and reflect the onset and development of the tumor [10]. In the last years, many researchers have demonstrated an increased interest in blood free amino acid profile and their potential use as biomarkers of cancer and other pathologies [1115]. Earlier studies showed that plasma amino acid levels alteration in cancer patients were closely related to cachexia and weight loss $[16,17]$. However, it has been observed that amino acids profile in cancer patients who also present cachexia is different from those malnourished patients with other pathologies [14]. In addition, changes in amino acid levels have also been observed in patients with normal weight and serum albumin values. It has also been described that alterations in amino acid levels occur in the first stages of the disease and there are no significant changes between the initial and subsequent stages [16]. In addition, different researchers have suggested the possibility of establishing a specific amino acid profile for each type of cancer. In fact, Miyagi et al. [2] distinguish two types of alterations in plasma amino acid levels in cancer patients: First, changes in a limited number of amino acids that reflect common metabolic changes in many types of cancer and second, changes in a high number of amino acids which represent specific metabolic characteristics to each tumor type. By other hand, the relationship between cancer and specific nutrients is also known and many epidemiological evidences have suggested that diet affects cancer risk and mortality. Thus, fat and fatty acid intake has been shown to have some associations with different types of cancer as lung, colon and breast cancer risk [18].

Breast cancer continues to be one of the most frequent and deadly pathologies in women despite the new strategies and therapies that have emerged in recent years, but they are not effective against all the breast cancer subtypes due to their genetic diversity $[19,20]$. Therefore, the knowledge of new biomarkers for early detection and new therapeutic targets is necessary. In this way, several researchers have analyzed the plasma amino acid profile in patients with breast cancer in order to find those amino acids which could be considered as earlier biomarkers. In the etiology of breast cancer, dietary factors have also been analyzed and especially the influence of dietary fat has been studied, but the underlying mechanisms remain unclear [21].

In the present work, we analyze the influence of normolipidic dietary fats [olive oil, sunflower oil and sunflower oil enriched with oleic acid] on serum amino acids in rats with experimentally-induced

${ }^{*}$ Correspondence to: José Manuel Martínez-Martos, Experimental and Clinical Physiopathology Research Group, Department of Health Sciences, School of Experimental and Health Sciences, University of Jaén, Campus Universitario Las Lagunillas, E-23071, Jaén, Spain

Key words: Breast cancer, $N$-methyl-N-nitrosourea, extra virgin olive oil, sunflower oil, biomarkers

Received: March 25, 2018; Accepted: April 14, 2018; Published: April 17, 2018 
mammary tumors. It will allow us to know the changes in circulating free amino acid levels in this animal model, if a specific profile of amino acids exits in this type of tumor, and if an external factor such as the type of dietary fat modifies this profile.

\section{Material and methods}

\section{Animals and treatment}

Seventy three female virgin Wistar rats $(149.4 \pm 2.7 \mathrm{~g}$ body weight $)$ were used in this work. The animals were provided from the animal house care of the University of Jaén, and maintained in an environment controlled under constant temperature $\left(25^{\circ} \mathrm{C}\right)$ with a 12-hr-light/12$\mathrm{hr}$ dark cycle. All animals were allowed access to water and food ad libitum. The experimental procedures for animal use and care were in accordance with the European Community Council directive (2010/63/ EU) and approved by the ethical committee of the University of Jaén. The rats were randomly divided into five groups. Four groups of 16 animals were injected intraperitoneally with three doses of $50 \mathrm{mg} / \mathrm{Kg}$ body weight of NMU at 50,80 and 110 days after birth. All rats were on estrus cycle during the first injection, verified by daily vaginal smears. Animals were fed with AIN-93M diets containing $4 \%$ of fat, constituted by extra virgin olive oil (EVOO dietary group), refined sunflower oil (SO dietary group) or refined sunflower oil enriched with $50 \%$ oleic acid (OAESO dietary group). The fourth dietary group received a commercial standard diet with $4 \%$ soybean oil (Musal and Chemical, Spain). Finally, a fifth group of 9 animals was considered as control and animals were injected intraperitoneally with the vehicle only and fed with the commercial diet. After 17 weeks of the first NMU injection, animals were sacrificed under equithesin anaesthesia $(2 \mathrm{ml} / \mathrm{kg}$ body weight), and a complete post-mortem examination was carried out. Blood samples were obtained in tubes without anticoagulants through the left cardiac ventricle and centrifuged ten minutes at $3000 \mathrm{~g}$ at $4{ }^{\circ} \mathrm{C}$ to obtain the serum. These samples were frozen and stored at $-80^{\circ} \mathrm{C}$, until use. The parameters of carcinogenesis and the histopathological characteristics of the different dietary groups have been previously described [21].

\section{Amino acids determination}

Amino acids content was assayed by HPLC coupled to a fluorescence detection system. Serum samples $(40 \mu \mathrm{L})$ were deproteinated by ultrafiltration through a 10,000 molecular weight cutoff filter. The deproteinated serum was precolumn derivatized with OPA reagent (o-phthaldialdehyde in borate buffer $\mathrm{pH} 9.5$ containing 3-mercaptopropionic acid) and injected through a refrigerated Perkin-Elmer Series 200 automatic sample injector into a $150 \times 3.9$ mm Waters Resolve $5 \mu$ C-18 column. The mobile phase consisted of: (A) $53 \mathrm{mM}$ sodium phosphate plus $1.2 \mathrm{mM}$ EDTA (pH 6.3), and (B) $50 / 50$ acetonitrile/water. A flow rate of $1 \mathrm{ml} / \mathrm{min}$ was maintained with a Perkin-Elmer Series 200 pump, using gradient of solvent A from 90 to $0 \%$ in $30 \mathrm{~min}$. The fluorescence detector (Perkin-Elmer Series 200a) was set at an excitation wavelength of $340 \mathrm{~nm}$ and an emission wavelength of $420 \mathrm{~nm}$. Data was processed with the TotalChrom WorkStation ver. 6.3.1 software from Perkin-Elmer. Concentrations were expressed as nmoles of amino acid per milliliter.

\section{Calculations}

Val, Leu, and Ile were summed as branched-chain amino acids (BCAAs). BCAAs plus the aromatic amino acids Tyr and Phe were summed as large neutral amino acids (LNAAs). BCAAs along with Phe, Met, Thr, Lys, and His were summed as essential amino acids
(EAAs). Ala, Gly, Ser, Gln, Arg, Tau, Gln, Glu, Asn, Asp, Orn and Cit were summed as nonessential amino acids (NEAAs). Arg, Orn, Lys and His were summed as basic amino acids (BAAs). The Phe:Tyr ratio was also calculated because it is typically lower when there is a deficit in dietary protein [22].

\section{Statistical analysis}

All values represent the mean of the individual determination \pm standard error of the mean (SEM). Data was analyzed by multiple analysis of variance (MANOVA) plus Newman-Keul's post-hoc test, using IBM Pass V.19 software. Values of $\mathrm{P}<0.05$ were considered significant.

\section{Results}

Free serum amino acid levels in healthy control animals and in animals with $\mathrm{NMU}$-induced breast cancer fed with different dietary fats (commercial, EVOO, SO and OAESO) are showed in Table 1. We did not find significant differences between groups for Tyr, Phe, Val, Met, Ile, Leu, His, Cit, GABA, BCAAs and LNAAs levels or the Phe:Tyr ratio. On the contrary, significant increases in serum Asp ( $\mathrm{p}<0.01)$, Gln $(\mathrm{p}<0.05)$, Gly $(\mathrm{p}<0.01)$, Ala $(\mathrm{p}<0.05)$, Thr $(\mathrm{p}<0.01), 1$-met-His $(\mathrm{p}<0.01)$, 3-met-His $(\mathrm{p}<0.01)$, Orn $(\mathrm{P}<0.01)$, Tau $(\mathrm{p}<0.01)$, NEAAs $(\mathrm{p}<0.05)$ and BAAs $(\mathrm{p}<0.05)$ levels were observed in animals with NMUinduced breast cancer fed with a commercial diet when compared with healthy control animals. Animals with NMU-induced breast cancer fed with EVOO showed also higher serum levels of Aad $(\mathrm{p}<0.05)$, Gln $(\mathrm{p}<0.05)$, Trp $(\mathrm{p}<0.01)$, Gly $(\mathrm{p}<0.01)$, Thr $(\mathrm{p}<0.01)$, Ala $(\mathrm{p}<0.01)$, 1 -met-His $(\mathrm{p}<0.01), 3$-met-His $(\mathrm{p}<0.01)$, Lys $(\mathrm{p}<0.01)$, Arg $(\mathrm{p}<0.01)$, Orn $(\mathrm{p}<0.01)$, Tau $(\mathrm{p}<0.01)$, EAAs $(\mathrm{p}<0.01)$, NEAAs $(\mathrm{p}<0.01)$ and BAA $(p<0.01)$ than animals of healthy control group. In the same way, animals with NMU-induced breast cancer fed with SO showed also higher serum levels of Aad $(\mathrm{p}<0.05)$, Asn $(\mathrm{p}<0.01)$, Ser $(\mathrm{p}<0.05)$, Trp $(\mathrm{p}<0.01)$, Gly $(\mathrm{p}<0.01)$, Thr $(\mathrm{p}<0.01)$, Ala $(\mathrm{p}<0.01), 1$-met-His $(\mathrm{p}<0.01)$, 3-met-His ( $p<0.01)$, Lys $(\mathrm{p}<0.05), \operatorname{Arg}(\mathrm{p}<0.01)$, Orn $(\mathrm{p}<0.01)$, NEAAs $(\mathrm{p}<0.01)$ and BAAs $(\mathrm{p}<0.01)$ than animals of healthy control group. On the contrary, animals with NMU-induced breast cancer fed with OAESO showed only higher serum levels of $\operatorname{Gln}(\mathrm{p}<0.05)$, Ala $(\mathrm{p}<0.05)$ and Orn $(\mathrm{p}<0.01)$, whereas Glu and Ser were significantly decreased $(\mathrm{p}<0.01$ and $\mathrm{p}<0.05$ respectively) when compared with the animals of healthy control group.

Regarding the dietary groups, a group of amino acids exist that increase with breast cancer and remain high when commercial, AOVE or SO fats are used, but dietary OAESO return their values to control levels. They are Gly $(\mathrm{p}<0.01)$, Thr $(\mathrm{p}<0.01), 1$-met-His $(\mathrm{p}<0.01)$, 3 -met-His $(\mathrm{p}<0.01)$, NEAAs $(\mathrm{p}<0.05)$ and BAAs $(\mathrm{p}<0.05)$. Similarly, Gln increases $(p<0.05)$ with breast cancer and remain high when commercial, AOVE or OAESO fats are used, but dietary SO return their values to control levels. Only serum Ala and Orn levels were significantly increased $(\mathrm{p}<0.05 ; \mathrm{p}<0.01$ respectively) in animals with NMU-induced breast cancer independently of the dietary fat used.

By other hand, a second group of amino acids did not were significantly modified in animals with breast cancer when a commercial diet was administrated, but significant changes were found when other dietary fats. Thus, serum Aad ( $\mathrm{p}<0.05$ or $\mathrm{p}<0.01)$, Trp $(\mathrm{p}<0.01)$, Lys $(\mathrm{p}<0.01)$ and $\operatorname{Arg}(\mathrm{p}<0.01)$ levels were significantly modified when EVOO and SO but not OAESO were used as dietary fats. In the same way, Glu, Asn and Ser as well as EAAs did not significantly change in animals with NMU-induced breast cancer fed with commercial diet, 
Table 1. Serum free amino acids in rats with breast cancer fed with the different dietary fats.

\begin{tabular}{|c|c|c|c|c|c|c|}
\hline \multirow{2}{*}{ Amino acid } & \multirow{2}{*}{ Abbreviation } & \multicolumn{5}{|c|}{ Experimental group ${ }^{a}$} \\
\hline & & Healthy control & Commercial & EVOO & SO & OAESO \\
\hline Aminoadipic acid & Aad & $185.1 \pm 6.8$ & $148.1 \pm 12.9$ & $181.3 \pm 8.3$ & $203.4 \pm 17.4$ & $215.6 \pm 19.9$ \\
\hline Aspartic acid & Asp & $4.5 \pm 0.4$ & $8.1 \pm 0.7$ & $6.4 \pm 0.3$ & $5.4 \pm 0.6$ & $3.2 \pm 0.5$ \\
\hline Glutamic acid & Glu & $50.4 \pm 8.7$ & $46.7 \pm 12.7$ & $49.9 \pm 6.1$ & $58.5 \pm 10.6$ & $27.8 \pm 7.9$ \\
\hline Asparagine & Asn & $164.7 \pm 18.0$ & $163.9 \pm 22.3$ & $169.1 \pm 12.6$ & $212.3 \pm 16.6$ & $153.6 \pm 13.4$ \\
\hline Glutamine & Gln & $4459.6 \pm 116.0$ & $5299.0 \pm 177.6$ & $5617.9 \pm 131.0$ & $4708.4 \pm 123.2$ & $5169.0 \pm 174.0$ \\
\hline Serine & Ser & $244.2 \pm 21.6$ & $225.5 \pm 26.5$ & $289.6 \pm 19.7$ & $323.0 \pm 22.8$ & $172.0 \pm 17.5$ \\
\hline Threonine & Thr & $306.03 \pm 24.56$ & $495.06 \pm 11.12$ & $589.46 \pm 29.0$ & $509.75 \pm 56.99$ & $355.91 \pm 37.04$ \\
\hline Tyrosine & Tyr & $52.7 \pm 7.4$ & $46.7 \pm 8.2$ & $48.1 \pm 4.3$ & $53.6 \pm 9.7$ & $50.0 \pm 5.8$ \\
\hline Tryptophan & Trp & $62.0 \pm 7.3$ & $63.1 \pm 6.2$ & $86.1 \pm 6.6$ & $84.0 \pm 7.0$ & $57.4 \pm 5.6$ \\
\hline Phenylalanine & Phe & $30.1 \pm 4.6$ & $30.9 \pm 4.3$ & $30.4 \pm 3.0$ & $30.1 \pm 6.6$ & $30.7 \pm 3.0$ \\
\hline Glycine & Gly & $410.6 \pm 23.5$ & $583.5 \pm 20.3$ & $676.5 \pm 28.1$ & $593.9 \pm 28.9$ & $439.3 \pm 26.6$ \\
\hline Alanine & Ala & $692.1 \pm 32.7$ & $814.1 \pm 28.7$ & $914.9 \pm 43.9$ & $812.9 \pm 42.2$ & $919.4 \pm 32.3$ \\
\hline Valine & Val & $136.1 \pm 9.4$ & $135.5 \pm 10.4$ & $134.8 \pm 9.3$ & $136.0 \pm 10.0$ & $135.3 \pm 10.3$ \\
\hline Methionine & Met & $45.5 \pm 5.8$ & $44.4 \pm 3.0$ & $47.8 \pm 9.9$ & $48.6 \pm 4.1$ & $46.2 \pm 6.0$ \\
\hline Isoleucine & Ile & $47.9 \pm 4.2$ & $48.1 \pm 4.9$ & $51.9 \pm 3.5$ & $50.8 \pm 6.0$ & $50.0 \pm 3.6$ \\
\hline Leucine & Lau & $441.8 \pm 38.7$ & $443.6 \pm 33.3$ & $431.5 \pm 34.3$ & $440.5 \pm 39.0$ & $447.5 \pm 29.0$ \\
\hline Histidine & His & $113.5 \pm 8.1$ & $116.6 \pm 9.7$ & $122.3 \pm 9.9$ & $121.1 \pm 9.3$ & $113.2 \pm 4.6$ \\
\hline 1-Methyl-histidine & 1-Met-His & $5.4 \pm 0.6$ & $24.9 \pm 3.9$ & $17.5 \pm 3.2$ & $25.0 \pm 5.0$ & $9.4 \pm 1.0$ \\
\hline 3-Methyl-histidine & 3-Met-His & $20.1 \pm 1.8$ & $44.6 \pm 1.4$ & $42.2 \pm 4.0$ & $44.9 \pm 6.9$ & $32.7 \pm 3.9$ \\
\hline Lysine & Lys & $798.0 \pm 45.7$ & $785.6 \pm 53.5$ & $1030.4 \pm 43.2$ & $1328.8 \pm 47.5$ & $787.7 \pm 48.0$ \\
\hline Arginine & Arg & $17.3 \pm 7.4$ & $16.2 \pm 5.6$ & $48.0 \pm 11.8$ & $69.1 \pm 16.8$ & $16.1 \pm 7.2$ \\
\hline Citrulline & Cit & $57.1 \pm 4.5$ & $57.5 \pm 7.9$ & $53.6 \pm 3.0$ & $51.2 \pm 8.0$ & $51.6 \pm 8.5$ \\
\hline Ornithine & Orn & $179.9 \pm 21.3$ & $289.5 \pm 16.0$ & $373.3 \pm 9.3$ & $371.8 \pm 32.7$ & $248.6 \pm 31.1$ \\
\hline$\gamma$-aminobutyric acid & GABA & $4.7 \pm 0.6$ & $4.3 \pm 0.5$ & $4.2 \pm 0.5$ & $4.5 \pm 0.6$ & $4.7 \pm 0.6$ \\
\hline Taurine & Tau & $656.3 \pm 20.9$ & $838.3 \pm 16.4$ & $886.1 \pm 32.3$ & $734.8 \pm 38.1$ & $607.2 \pm 39.1$ \\
\hline EAAs & Essential amino acids & $2330.3 \pm 131.2$ & $2252.5 \pm 197.3$ & $3112.8 \pm 144.2$ & $2375.1 \pm 200.8$ & $2533.6 \pm 208.9$ \\
\hline NEAAs & $\begin{array}{l}\text { Non-essential amino } \\
\text { acids }\end{array}$ & $6636.7 \pm 152.0$ & $7818.0 \pm 223.4$ & $8432.4 \pm 204.7$ & $7425.8 \pm 538.2$ & $7236.6 \pm 304.3$ \\
\hline BCAAs & $\begin{array}{l}\text { Branched chain amino } \\
\text { acids }\end{array}$ & $625.9 \pm 54.9$ & $627.4 \pm 51.3$ & $639.3 \pm 46.7$ & $629.4 \pm 50.9$ & $622.9 \pm 41.5$ \\
\hline LNAAs & $\begin{array}{l}\text { Large neutral amino } \\
\text { acids }\end{array}$ & $775.9 \pm 70.0$ & $778.2 \pm 69.3$ & $774.0 \pm 54.7$ & $760.8 \pm 49.0$ & $748.2 \pm 52.9$ \\
\hline BAAs & Basic amino acids & $1038.0 \pm 86.2$ & $1114.9 \pm 57.9$ & $1469.5 \pm 165.2$ & $1931.0 \pm 281.7$ & $1155.8 \pm 115.7$ \\
\hline
\end{tabular}

but change in a different degree and without a consistent profile when compared with the other dietary groups analyzed.

Finally, serum Asp level was significantly higher $(\mathrm{p}<0.01)$ in animals with NMU-induced breast cancer fed with a commercial diet when compared with healthy animals, although animals with breast cancer fed with EVOO, SO or OAESO did not change their serum Asp levels when compared with animals of healthy group. Similarly, serum Tau level was significantly higher $(\mathrm{p}<0.01)$ in animals with breast cancer fed with commercial and EVOO diet, but no changes were detected in serum Tau level in animals with breast cancer fed with SO and OAESO when compared with the animals of the control healthy group.

\section{Discussion}

Circulating free amino acid levels are the reflection of all the factors influencing the total flux of amino acids in the organism [6]. In healthy populations, amino acids are regulated by a continual cycling of protein synthesis and degradation to maintain a neutral protein balance [1]. But amino acids also act as metabolic regulators [2,3] of gene expression and cell growth and proliferation [3-5], which may be involved in tumoral processes. In fact, the presence of a tumour has also been associated with an alteration of protein metabolism which is mediated by the tumour itself and characterized by an increased protein turnover [6].
It has been also accepted that the availability of free amino acids is often reduced in cancer patients due to malnutrition but also by the increase in the amino acid demand as a consequence of the presence of the tumor; in fact, proteins are a reservoir of metabolic energy that may be seriously reduced during tumor growth [10]. For their growth, tumors require EAAs, in particular, Gln, Gly and Asp (necessary for the purine and pyrimidine synthesis) and Ser (necessary for the synthesis of membrane lipid compounds). This demand for amino acids generally leads to a gradual loss of muscle mass [23,24], which in turn causes a decrease in the availability of amino acids, especially EAAs. However, all types of tumors have not the same demand for amino acids. In fact, the analysis of plasma amino acid profile in patient with cancer showed heterogeneous results and three key variables have been described: The tumor type, the tumor grade and the nutritional status of the patient. Also, several authors had considered that the most important changes in plasma amino acid profile happened in patients with metastases, that is, in the more advanced stages of the tumor and in those patients whose also present anorexia, malnutrition and progressive weight loss [2527]. However, it has been recently observed that the changes in plasma amino acid profile in patients with cancer and caquexia are different to those detected in malnourished patients with other pathologies. In fact, changes in amino acid levels have also been detected in patients with normal weight and serum levels of albumin [16]. In addition to these 
factors, others such as the technique used to evaluate the metabolism of amino acids [28,29], the use of enteral or parenteral nutrition [26] or even the hormonal status $[6,10]$ can affect the circulating free amino acid profile.

All these evidences have suggested the possible role of amino acids as biomarkers of cancer. In fact, there are many studies focused on the role of circulating free amino acids as biomarkers of alterations in protein metabolism associated with cancer [30,31]. In addition, the description of different amino acid profiles for specific types of cancer has suggested that the metabolic alterations of each type of tumor determine its own plasma amino acid profile; therefore, the possibility that each type of cancer can promote specific amino acid profile has suggested the important role of amino acids in the diagnosis of this pathology $[6,11-13,15,16,32,33]$.

In the first studies in which the amino acid profile was analyzed in different tumors, Ching et al. [26] described significant deficiencies of Gly and Thr in patients with tumors located in the neck and head. The authors also observed differences in both EAAs and NEAAs level in patients with chemotherapy treatment. Bennegard et al. [34] also described in cancer patients with weight losses of more than $7 \%$, significant decreases in Ala, Cit, Glu/Gln, His, Pro, Ser and Thr levels as well as a significant increase in Phe. These authors established that certain amino acids considered individually could have a specific metabolism in patients with cancer and could even be modified depending on the malignancy of the tumor even no changes were induced in the rest of the amino acids. Miyagi et al. [2] also studied the possibility of using the plasma amino acid profile in the diagnosis of different tumors and determined the existence of significant alterations between cancer patients and healthy controls. In addition, they also found that some of these differences reflected common metabolic changes in many types of cancer and some of these changes observed in patients with tumors were maintained from the earliest stages.

In patients with breast cancer, the results are also heterogeneous. Kubota et al. [30] described a decrease in Cys and Gln levels and an increase in Ala, Arg and Thr levels in women with breast cancer, while Cascino et al. [35] described increases in Orn, Glu and free Trp levels. More recent studies also show great heterogeneity in their results when compared women with breast cancer and healthy volunteers $[2,3,36,37]$. Miyagi et al. [2] analyzed the plasma amino acid profile in 196 patients with breast cancer (55\% of them in the first stages) and 976 healthy controls. They observed significant changes in the amino acid profile between cancer patients and the control group. Significant higher plasma levels of Thr, Pro, Ser, Gly, Ala and Orn and significantly lower for Gln, Trp, Phe and Tyr were described in women with breast cancer. These results were similar to those found in other previous studies. Lai et al. [10] also found decreases in His and Gln and increases in Pro and Ala. Gu et al. [16] analyzing 28 patients with breast cancer (most in early stages) and 137 healthy ones found that the amino acid profile was different between breast cancer patients and controls, reporting that Thr and Arg levels were elevated and Asp, Glu, Gly, His and Pro levels were decreased. The authors also described that Ala induces the proliferation of breast cancer cells. Therefore, Ala was proposed as a possible marker for the diagnosis of cancer.

Our results showed a large group of amino acids that did not modify their plasma concentration after the induction of tumors (Glu, Ser, Thr, Tyr, Phe, Val, Met, Iso, Leu, His, Lys and EAAs). These data are in accordance with those described by Proenza et al. [6] in women with breast cancer. However, other group of amino acids showed changes in animals with induced breast cancer but no changes have been described in women. These differences may be due to differences in metabolism between species. Some of these amino acids are Gln, Asp, Ala and Gly, which levels were significantly increased in animals with breast cancer in relation to healthy controls. Some authors have considered, as previously mentioned, that these amino acids are essential for tumor growth.

On the other hand, this paper also analyzes the influence of an external factor such as the type of dietary fat on circulating free amino acid levels. In most of human studies, patients and controls groups are usually homogenized in terms of cancer related factors such as smoking, weight, age and gender; but it is difficult to control the effects of other factors also involved in cancer as diet. Our results showed significant changes in amino acid levels in relation with the dietary fat. Ala and Orn levels were significant increased in animal with induced breast cancer and were the only amino acids not influenced by the diet. Therefore, these amino acids could be considered as potential biomarkers because they are not affected by external factors. These data are also in accordance with the roles of Orn in the inmune response and Ala as inductor of cellular proliferation previously described [2].

It must be also take into account that many authors have considered that the effect of breast cancer in the availability of amino acids is lesser than other types of tumors such as lung cancer [6]. These data may reflect, by one hand, a greater resistance to the effects of the tumor and, on the other hand, a lower rate of breast cancer to induce metabolic stress. In any case, these results may justify the relationship between circulating free amino acid levels and cancer. However, to establish a specific amino acid profile for each type of cancer and use it as biomarker, it could be necessary to consider that many of the changes observed in amino acids may be masked by external factors such as the type of dietary fat.

\section{Disclosure statement}

No conflicts of interest, financial or otherwise, are declared by the authors.

\section{Funding details}

This work was supported by the Junta de Andalucía under Grant PAIDI CTS-1039.

\section{References}

1. Barnes T, Bell K, DiSebastiano KM, Vance V, Hanning R, et al. (2014) Plasma amino acid profiles of breast cancer patients early in the trajectory of the disease differ from healthy comparison groups. Appl Physiol Nutr Metab 39: 740-744. [Crossref]

2. Miyagi Y, Higashiyama M, Gochi A, Akaike M, Ishikawa T, et al. (2011) Plasma free amino acid profiling of five types of cancer patients and its application for early detection. PLoS One 6: e24143. [Crossref]

3. Nagata C, Wada K, Tsuji M, Hayashi M, Takeda N, et al. (2014) Plasma amino acid profiles are associated with biomarkers of breast cancer risk in premenopausal Japanese women. Cancer Causes Control 25: 143-149. [Crossref]

4. Marshall S (2006) Role of insulin, adipocyte hormones, and nutrient-sensing pathways in regulating fuel metabolism and energy homeostasis: a nutritional perspective of diabetes, obesity, and cancer. Sci STKE 2006: re7. [Crossref]

5. Wu G (2009) Amino acids: metabolism, functions, and nutrition. Amino Acids 37: 1-17 [Crossref]

6. Proenza AM, Oliver J, Palou A, Roca P (2003) Breast and lung cancer are associated with a decrease in blood cell amino acid content. J Nutr Biochem 14: 133-138. [Crossref]

7. Hadi NI, Jamal Q (2015) "OMIC" tumor markers for breast cancer: A review. Pak J Med Sci 31: 1256-1262. [Crossref]

8. Altman BJ, Dang CV (2012) Normal and cancer cell metabolism: lymphocytes and lymphoma. FEBS J 279: 2598-609. [Crossref] 
9. Hiller K, Metallo CM (2013) Profiling metabolic networks to study cancer metabolism. Curr Opin Biotechnol 24: 60-68. [Crossref]

10. Lai HS, Lee JC, Lee PH, Wang ST, Chen WJ (2005) Plasma free amino acid profile in cancer patients. Semin Cancer Biol 15: 267-276. [Crossref]

11. Delgado-Povedano MM, Calderon-Santiago M, Priego-Capote F, Luque de Castro MD (2016) Study of sample preparation for quantitative analysis of amino acids in human sweat by liquid chromatography-tandem mass spectrometry. Talanta 146: 310-317. [Crossref]

12. Bi X, Henry CJ (2017) Plasma-free amino acid profiles are predictors of cancer and diabetes development. Nutr Diabetes 7: e249. [Crossref]

13. Poschke I, Mao Y, Kiessling R, de Boniface J (2013) Tumor-dependent increase of serum amino acid levels in breast cancer patients has diagnostic potential and correlates with molecular tumor subtypes. J Transl Med 11: 290. [Crossref]

14. Siminska E, Koba M (2016) Amino acid profiling as a method of discovering biomarkers for early diagnosis of cancer. Amino Acids 48: 1339-1345. [Crossref]

15. Wu G (2013) Functional amino acids in nutrition and health. Amino Acids 45: 407-411. [Crossref]

16. Gu Y, Chen T, Fu S, Sun X, Wang L, et al. (2015) Perioperative dynamics and significance of amino acid profiles in patients with cancer. J Transl Med 13: 35. [Crossref]

17. Argiles JM, Busquets S, Stemmler B, Lopez-Soriano FJ (2014) Cancer cachexia: understanding the molecular basis. Nat Rev Cancer 14: 754-762. [Crossref]

18. Kerr J, Anderson C, Lippman SM (2017) Physical activity, sedentary behaviour, diet, and cancer: an update and emerging new evidence. Lancet Oncol 18: e457-e471. [Crossref]

19. Geck RC, Toker A (2016) Nonessential amino acid metabolism in breast cancer. $A d v$ Biol Regul 62: 11-17. [Crossref]

20. Siegel RL, Miller KD, Jemal A (2015) Cancer statistics, 2015. CA Cancer J Clin 65 5-29. [Crossref]

21. Ruiz-Sanjuan MD, Martinez-Martos JM, Carrera-Gonzalez MP, Mayas MD, Garcia MJ, et al. (2015) Normolipidic dietary fat modifies circulating Renin-Angiotensin system-regulating aminopeptidase activities in rat with breast cancer. Integr Cancer Ther 14: 149-155. [Crossref]

22. Antener I, Tonney G, Verwilghen AM, Mauron J (1981) Biochemical study of malnutrition. Part IV. Determination of amino acids in the serum, erythrocytes, urine and stool ultrafiltrates. Int J Vitam Nutr Res 51: 64-78. [Crossref]

23. Levin L, Gevers W (1981) Metabolic alterations in cancer. Part II. Protein and fat metabolism. S Afr Med J 59: 553-556. [Crossref]
24. Argiles JM, Azcon-Bieto J (1988) The metabolic environment of cancer. Mol Cell Biochem 81: 3-17. [Crossref]

25. Clarke EF, Lewis AM, Waterhouse C (1978) Peripheral amino acid levels in patients with cancer. Cancer 42: 2909-2913. [Crossref]

26. Ching N, Grossi C, Jham G, Angers J, Zurawinsky H, et al. (1984) Plasma amino acid and serum unesterified fatty acid deficits and the effect of nutritional support in chemotherapy treatment. Surgery 95: 730-738. [Crossref]

27. Norton JA, Gorschboth CM, Wesley RA, Burt ME, Brennan MF (1985) Fasting plasma amino acid levels in cancer patients. Cancer 56: 1181-1186. [Crossref]

28. Millward DJ, Fereday A, Gibson NR, Pacy PJ (2000) Human adult amino acid requirements: [1-13C]leucine balance evaluation of the efficiency of utilization and apparent requirements for wheat protein and lysine compared with those for milk protein in healthy adults. Am J Clin Nutr 72: 112-121. [Crossref]

29. Bos C, Gaudichon C, Tome D (2002) Isotopic studies of protein and amino acid requirements. Curr Opin Clin Nutr Metab Care 5: 55-61. [Crossref]

30. Kubota A, Meguid MM, Hitch DC (1992) Amino acid profiles correlate diagnostically with organ site in three kinds of malignant tumors. Cancer 69: 2343-2348. [Crossref]

31. Rossi Fanelli F, Cangiano C, Muscaritoli M, Conversano L, Torelli GF, et al. (1995) Tumor-induced changes in host metabolism: a possible marker of neoplastic disease. Nutrition 11: 595-600. [Crossref]

32. Kim HJ, Jang SH, Ryu JS, Lee JE, Kim YC, et al. (2015) The performance of a nove amino acid multivariate index for detecting lung cancer: A case control study in Korea. Lung Cancer 90: 522-527. [Crossref]

33. Shingyoji M, Iizasa T, Higashiyama M, Imamura F, Saruki N, et al. (2013) The significance and robustness of a plasma free amino acid (PFAA) profile-based multiplex function for detecting lung cancer. BMC Cancer 13: 77. [Crossref]

34. Bennegard K, Lindmark L, Eden E, Svaninger G, Lundholm K (1984) Flux of amino acids across the leg in weight-losing cancer patients. Cancer Res 44: 386-393. [Crossref]

35. Cascino A, Muscaritoli M, Cangiano C, Conversano L, Laviano A, et al. (1995) Plasma amino acid imbalance in patients with lung and breast cancer. Anticancer Res 15: 507510. [Crossref]

36. Chen Y, Zhang R, Song Y, He J, Sun J, et al. (2009) RRLC-MS/MS-based metabonomics combined with in-depth analysis of metabolic correlation network: finding potential biomarkers for breast cancer. Analyst 134: 2003-2011. [Crossref]

37. Slupsky CM, Steed H, Wells TH, Dabbs K, Schepansky A, et al. (2010) Urine metabolite analysis offers potential early diagnosis of ovarian and breast cancers. Clin Cancer Res 16: 5835-5841. [Crossref]

Copyright: (C2018 Ramírez-Expósito MJ. This is an open-access article distributed under the terms of the Creative Commons Attribution License, which permits unrestricted use, distribution, and reproduction in any medium, provided the original author and source are credited. 